\title{
Preparation of carbon nanotubes via chemical technique (modified Staudenmaier method)
}

\begin{abstract}
The chemical method is one of the most promising routes for the production of multi-walled carbon nanotubes. In this study, multi-walled carbon nanotubes (MWCNTs) were successfully synthesized by the modified Staudenmaier method. MWCNTs were synthesized using graphite powder at room temperature. This process does not utilize any metal catalyst. The prepared multi-walled carbon nanotubes were characterized for their crystalline, structural and thermal stability properties. The structural, crystalline and morphological characterization of The produced MWCNTs were performed by using scanning electron microscopy(SEM), transmission electron microscopy(TEM), X-ray diffraction (XRD), thermogravimetric analysis(TGA), Raman analysis, and atomic force microscopy( AFM). Crystallinity and graphitization degree was observed for the produced MWCNTs have a diameter of $13.1 \mathrm{~nm}$. The yield is approximately $10 \%$ of the carbon source (graphite). Moreover, the obtained MWCNTs have high thermal stability and oxidation resistance lower than $401.80{ }^{\circ} \mathrm{C}$. The present approach provides a simple low cost and easy method of MWCNTs synthesis.
\end{abstract}

Keyword: Multi-walled carbon nanotubes; Modified staudenmaier; Graphite; Diameter; Yield; CNTs 